\title{
Uninterrupted Transmission System for Realizing High Flexibility in Design of Angular Velocity Ratio*
}

\author{
Jungchul $\mathrm{KANG}^{* *}$ and Masaharu KOMORI** \\ **Department of Mechanical Engineering and Science, Kyoto University, \\ Yoshidahonmachi, Sakyo-ku, Kyoto-shi, Kyoto 606-8501, Japan \\ E-mail: komorim@me.kyoto-u.ac.jp
}

\begin{abstract}
Geared transmissions are used in a variety of mechanical devices to realize a wide range of speeds or torques. However, the transmission of power and motion in a geared transmission is interrupted when the working gear pairs are changed to vary the velocity ratio. In order to solve this problem, an uninterrupted transmission system was proposed in the previous report that can change the velocity ratio while transmitting power and rotation from the input shaft to the output shaft. However, in the uninterrupted transmission system, there are some cases in which it is difficult to design a suitable noncircular gear based on the combination of angular velocity ratios. In addition, since there is a design constraint that must be satisfied between the angular velocity ratio and the number of teeth on the clutch, the gears and clutches cannot be designed independently. To solve this problem, a new type of uninterrupted transmission system that enables the design of a suitable noncircular gear for various combinations of angular velocity ratios is proposed in this report. Furthermore, in order to avoid the constraint between the angular velocity ratio and the number of teeth on the clutch, a new transition method is proposed. An experimental device using the novel uninterrupted transmission system is constructed, and the effectiveness of the proposed system is verified by experiment.
\end{abstract}

Key words: Transmission, Gear, Velocity Ratio Change, Mechanism, Machine Element

\section{Introduction}

Mechanical devices are required to realize a wide range of speeds or torques, but their ranges are limited due to the characteristics of the engines or motors. In such cases, it is possible to expand the ranges of speeds and torques by using transmissions because they can change the velocity ratio (reduction ratio or angular velocity ratio) between the input shaft and output shaft. Currently, geared transmissions are widely used in industrial fields. Geared transmissions have advantages in their power transmission efficiency and large torque transmission capacity. Therefore, there have been many studies ${ }^{(1)-(3)}$ on mechanisms and controls of geared transmissions. However, in geared transmissions, it is necessary to switch the working gear pairs when the velocity ratio is changed. In this process, the power transmission between the input shaft and output shaft is interrupted. Thus, it is impossible to change the velocity ratio while maintaining the driving force. Also, in the field of industrial machinery, precise and accurate positioning is often required. In these applications, the 
rotational angle must be transmitted precisely from the input shaft to the output shaft even during the velocity ratio changing process, but this is impossible in conventional geared transmissions.

To solve these problems, Komori et al. ${ }^{(4)-(7)}$ proposed an uninterrupted transmission system using noncircular gears in the previous reports. This transmission system can transmit the torque and rotational angle precisely from the input shaft to the output shaft by using noncircular gears even during the velocity ratio changing process. However, in the uninterrupted transmission system, there are some cases in which it is difficult to design a suitable noncircular gear for this transmission system based on the combination of angular velocity ratios. In these cases, a smooth change of the velocity ratio cannot be achieved. Furthermore, in the uninterrupted transmission system, there is a design constraint to be satisfied between the angular velocity ratio and the number of teeth on the clutch used in the transmission system. Thus, the gears and clutches cannot be designed independently. To solve this problem, this report proposes a novel uninterrupted transmission system that enables the design of a suitable noncircular gear for various combinations of angular velocity ratios. Moreover, in order to avoid the constraint between the angular velocity ratio and the number of teeth on the clutch, a new transition method is proposed that can allow the independent design of the clutch and gear. An experimental device of the new type of uninterrupted transmission system with the new velocity ratio changing method is constructed, and its effectiveness is verified by experiment.

\section{Uninterrupted transmission system}

Figure 1 shows the structure of the uninterrupted transmission system proposed in the previous reports ${ }^{(4)-(7)}$. It is composed of an input shaft, an output shaft, circular gear pairs $\mathrm{A}$ and $B$, noncircular gear pair $C$, and clutches $T_{A}, T_{B}$, and $T_{C}$. Input gears $A_{i}, B_{i}$, and $C_{i}$ are fixed to the input shaft, and output gears $A_{0}, B_{0}$, and $C_{o}$ are supported rotatably by the output shaft via bearings. Output gears $\mathrm{A}_{o}, \mathrm{~B}_{\mathrm{o}}$, and $\mathrm{C}_{\mathrm{o}}$ rotate with the output shaft if clutches $\mathrm{T}_{\mathrm{A}}, \mathrm{T}_{\mathrm{B}}$, and $\mathrm{T}_{\mathrm{C}}$ are engaged, and the output gear can rotate independently of the output shaft if the clutch is disengaged.

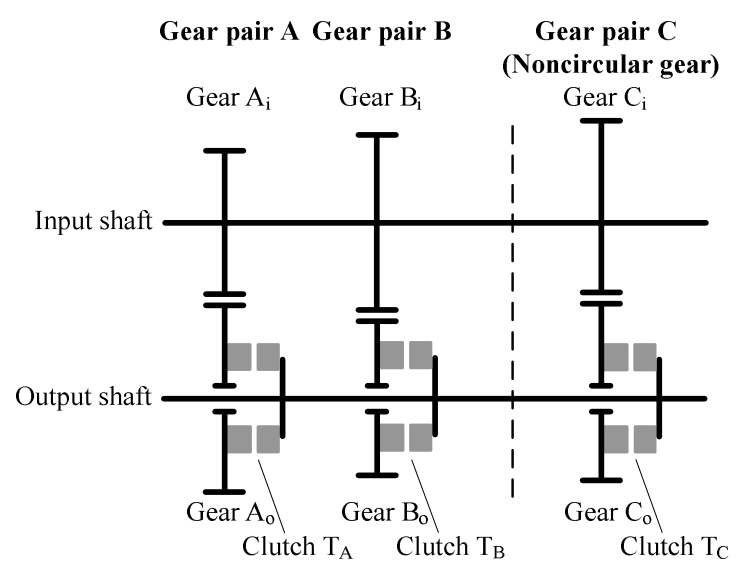

Fig. 1 Structure of uninterrupted transmission system

Suppose that the angular velocity ratio of gear pair $\mathrm{A}$ is $r_{A}$, and that of gear pair $\mathrm{B}$ is $r_{B}$. As shown in Fig. 2, the pitch curve of gear pair $\mathrm{C}$ consists of section a (which is the same as the pitch circle of gear pair A), section b (which is the same as the pitch circle of 
gear pair B), and sections s and $\mathrm{t}$ (which connect smoothly between sections a and $\mathrm{b}$ ). The angular velocity ratio is determined by the ratio of the distances between the contact point of the two pitch curves and the rotational center of each gear. As shown in Fig. 2(a), when noncircular gear pair $\mathrm{C}$ meshes at section a, the angular velocity ratio of gear pair $\mathrm{C}$ becomes the same as that of gear pair A because the meshing conditions of gear pair $\mathrm{C}$ and gear pair A are the same. On the other hand, as shown in Fig. 2(b), the meshing condition of gear pair $\mathrm{C}$ is the same as that of gear pair $\mathrm{B}$ when it meshes at section $\mathrm{b}$; then, the angular velocity ratio of gear pair $\mathrm{C}$ becomes the same as that of gear pair $\mathrm{B}$. If gear pair $\mathrm{C}$ rotates in the direction shown by the arrow in Fig. 2(a), after the meshing in section a is finished and gear pair $\mathrm{C}$ begins meshing in section $\mathrm{s}$, the angular velocity ratio changes continuously from that of gear pair A to that of gear pair B according to the rotation. In contrast, the angular velocity ratio changes from that of gear pair B to that of gear pair A when it meshes at section $\mathrm{t}$.

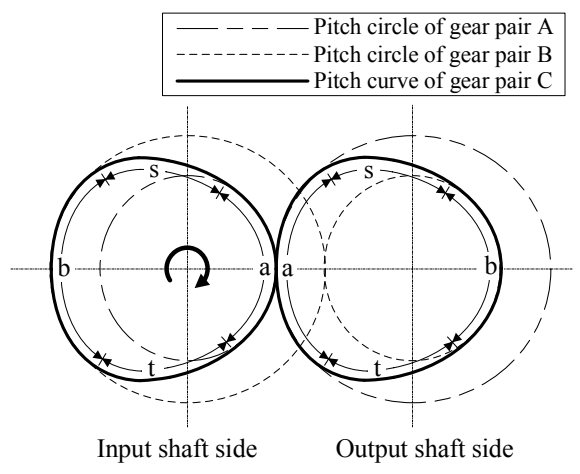

(a) The first ratio condition

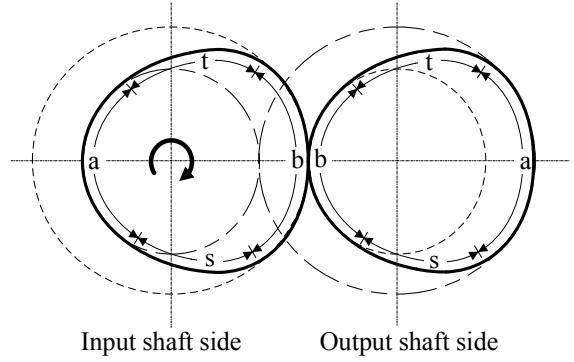

(b) The second ratio condition

Fig. 2 Pitch curves of noncircular gear pair $\mathrm{C}$

The velocity ratio changing process for the uninterrupted transmission system is shown in Fig. 3. Consider the case of a velocity ratio change from the first ratio condition (angular velocity ratio is $r_{A}$ ), where only clutch $\mathrm{T}_{\mathrm{A}}$ is engaged and clutches $\mathrm{T}_{\mathrm{B}}$ and $\mathrm{T}_{\mathrm{C}}$ are disengaged, to the second ratio (angular velocity ratio is $r_{B}$ ), where only clutch $\mathrm{T}_{\mathrm{B}}$ is engaged and the other clutches are disengaged. Since noncircular gear $C_{i}$ is fixed to the input shaft and noncircular gear pair $\mathrm{C}$ keeps rotating, the angular velocity ratio of gear pair $\mathrm{C}$ changes based on the rotational angle, as shown by the dashed curve in the lower part of Fig. 3. Therefore, at a certain time, noncircular gear pair $\mathrm{C}$ begins meshing in section a. At this time, clutch $\mathrm{T}_{\mathrm{C}}$ becomes engaged. Because the angular velocity ratio of noncircular gear pair $\mathrm{C}$ is the same as that of gear pair $\mathrm{A}$ in this state, output gears $\mathrm{C}_{\mathrm{o}}$ and $\mathrm{A}_{\mathrm{o}}$ rotate at the same rotational speed. Therefore, even if clutch $T_{C}$ and clutch $T_{A}$ are engaged simultaneously, the input and output shafts can rotate. In this state, both gear pairs $\mathrm{A}$ and $\mathrm{C}$ transmit the rotation.

Next, clutch $\mathrm{T}_{\mathrm{A}}$ becomes disengaged while noncircular gear pair $\mathrm{C}$ keeps meshing in section a. After this process, only noncircular gear pair $\mathrm{C}$ transmits the rotation. Furthermore, when noncircular gear pair $\mathrm{C}$ begins meshing in section $\mathrm{s}$, the angular velocity ratio between the input shaft and the output shaft changes according to the rotation of noncircular gear pair $\mathrm{C}$, and then the transition from the first ratio condition to the second ratio condition occurs.

Next, noncircular gear pair $C$ begins meshing in section $b$. At this time, clutch $T_{B}$ becomes engaged while clutch $T_{C}$ is still engaged. Although both clutches $T_{C}$ and $T_{B}$ are engaged, they can rotate because the angular velocity ratio of noncircular gear pair $\mathrm{C}$ is the same as that of gear pair B in this state. In this condition, both gear pair B and noncircular 
gear pair $\mathrm{C}$ transmit the rotation.

Then, clutch $\mathrm{T}_{\mathrm{C}}$ becomes disengaged before the meshing position of gear pair $\mathrm{C}$ changes from section $b$ to section $t$. After this process, only clutch $T_{B}$ is engaged, and only gear pair B is connected to the input and output shafts; in other words, it becomes the second ratio condition. Consequently, the velocity ratio change from the first ratio condition to the second ratio condition is completed.

In the uninterrupted transmission system, the noncircular gear transmits the torque and rotation while changing the angular velocity ratio. Therefore, it is possible to transmit the power and precise rotational angle even during the velocity ratio changing process, which is impossible in a conventional geared transmission.

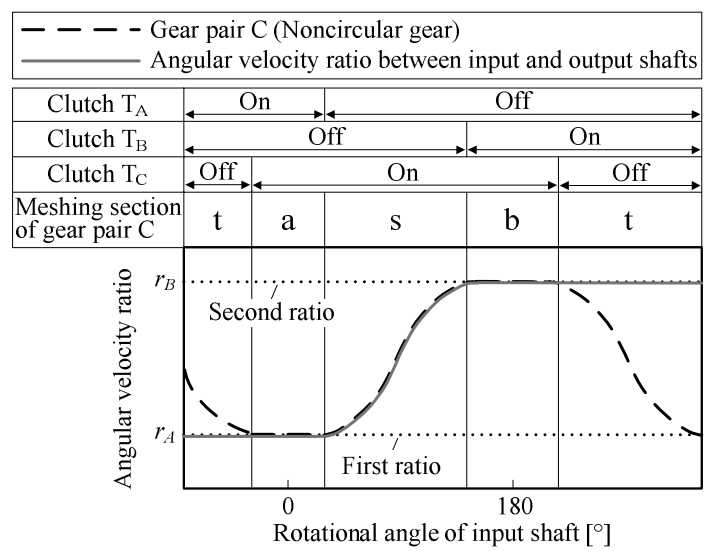

Fig. 3 Process to change the angular velocity ratio from the first ratio to the second ratio

\section{Uninterrupted transmission system for realizing various combinations of angular velocity ratios}

\subsection{Problems of the conventional uninterrupted transmission system}

Since various functions are required of mechanical devices, it is necessary to realize a variety of angular velocity ratios in the transmission systems used in these devices. However, in the conventional uninterrupted transmission system, problems occur in some cases depending on the combination of angular velocity ratios. This problem is explained here.

The tooth number ratio for a noncircular gear must be a natural number (e.g., 1 in many cases). Here, the tooth number ratio of the noncircular gear is called the average angular velocity ratio of the noncircular gear. For example, the average angular velocity ratio is 1 if the tooth number ratio of the noncircular gear is 1 . Take the case in which the uninterrupted transmission system is required to realize angular velocity ratios of $0.7\left(r_{A}\right)$ and $0.9\left(r_{B}\right)$. Figure 4(a) shows a schematic diagram of the angular velocity ratio of the noncircular gear for the uninterrupted transmission system corresponding to this condition. Sections $\mathrm{a}$ and $\mathrm{b}$ are constant angular velocity ratio sections. The angular velocity ratio is 0.7 in section a and 0.9 in section $b$. However, the values of the angular velocity ratios are larger in sections $s$ and $t$ than in sections $a$ and $b$. This condition is different from the ideal angular velocity ratio curve shown in Fig. 3.

The angular velocity ratios mentioned in this example are 0.7 and 0.9 , and both are less than 1. Therefore, sections that have angular velocity ratios larger than 1 are necessary because the average angular velocity ratio of the noncircular gear must be 1 . This is the reason why the angular velocity ratios are large at sections $\mathrm{s}$ and $\mathrm{t}$. When the angular velocity ratio change from 0.7 to 0.9 is carried out using the noncircular gear with the angular velocity ratio curve shown in Fig. 4(a) in the uninterrupted transmission system, 
sections $\mathrm{a}, \mathrm{s}$, and $\mathrm{b}$ are used. In this process, the angular velocity ratio is 0.7 in the initial state, it temporarily becomes 1.3 in the next step, and then it decreases again to 0.9 . When the angular velocity ratio changes in this manner, the rotational speed of the output shaft increases and decreases during the velocity ratio changing process. This situation is not desirable for many mechanical devices. The same problem occurs if the angular velocity ratio required for the uninterrupted transmission system is between a certain integer $n$ and $n+1$ because the average angular velocity ratio of the noncircular gear must be a natural number. For example, Fig. 4(b) shows another case, where angular velocity ratios of 1.2 and 1.3 are required for the uninterrupted transmission system, and both angular velocity ratios are between 1 and 2 . In sections $s$ and $t$, the angular velocity ratio must become lower than 1 , and the same problem mentioned above occurs.

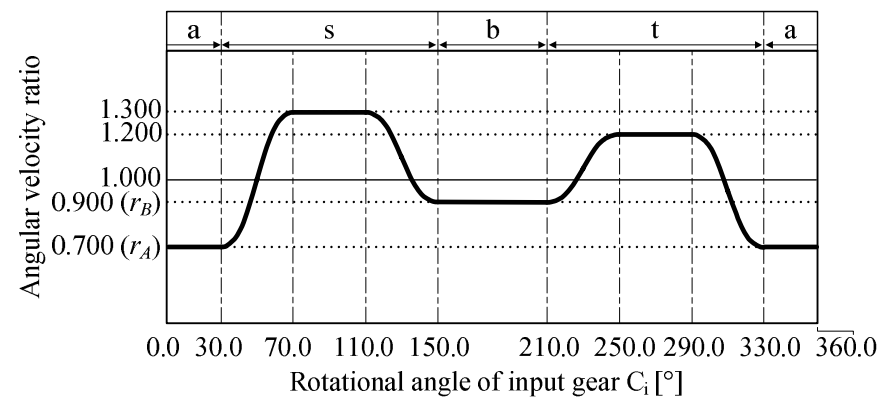

(a) The values of $r_{A}$ and $r_{B}$ are both less than 1

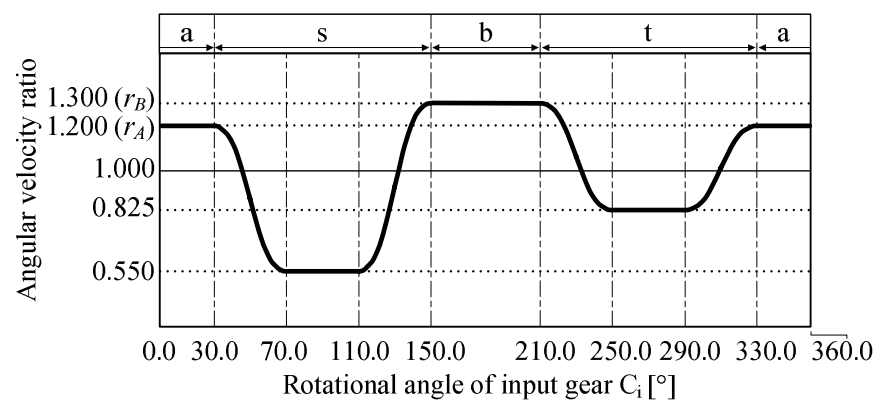

(b) The values of $r_{A}$ and $r_{B}$ are both larger than 1

Fig. 4 Schematic diagram of angular velocity ratio curve of noncircular gear pair C

The abovementioned problem does not occur if a certain natural number is sandwiched between the angular velocity ratios required for the uninterrupted transmission system. However, even in this case, another type of problem still remains. For example, take the case in which angular velocity ratios of 0.5 and 1.1 are required for the uninterrupted transmission system. Figure 5 shows a schematic diagram of the angular velocity ratio curve of the noncircular gear corresponding to this condition. In sections s and t, the angular velocity ratio changes smoothly between 0.5 and 1.1. Thus, the problem shown in Fig. 4 does not occur here. However, the range of section a (the range of rotational angle of noncircular input gear $\mathrm{C}_{\mathrm{i}}$ corresponding to section a), where the angular velocity ratio is 0.5 , is short. On the other hand, the range of section $b$, where the angular velocity ratio is 1.1 , is long. The difference between the angular velocity ratio of 0.5 and the average angular velocity ratio of 1 is large, but the difference between 1.1 and 1 is small. For this reason, in order to make the average angular velocity ratio of the noncircular gear 1 , it is necessary to make section a short and section $b$ long because the difference in the angular velocity ratio from the average angular velocity ratio is large in section a and small in section $b$. This is 
the reason why the range of section $b$ is longer than that of section $a$. In the uninterrupted transmission system, it is desirable in many cases that sections a and b (where the angular velocity ratio is constant) have almost the same length since the clutch engagement and disengagement operations are performed in these sections. Consequently, the situation in Fig. 5 should be improved.

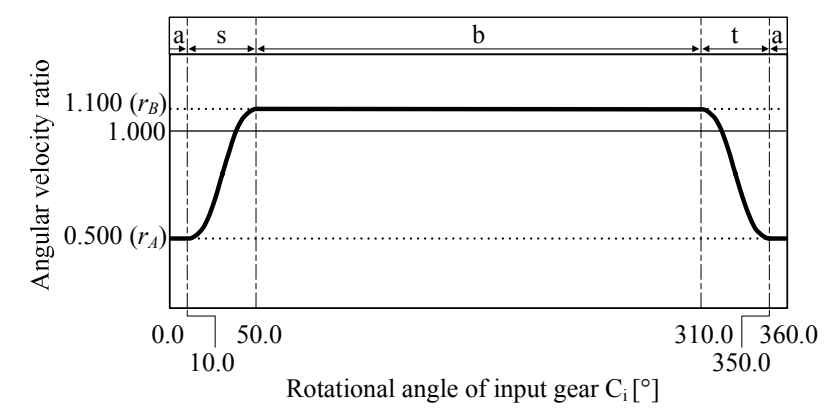

Fig. 5 Schematic diagram of angular velocity ratio curve of noncircular gear pair $\mathrm{C}$ when $r_{A}$ is 0.5 and $r_{B}$ is 1.1

\subsection{Uninterrupted transmission system applicable for various combinations of angular velocity ratios}

As mentioned in the previous section, in the conventional uninterrupted transmission system, it is difficult to realize a suitable noncircular gear in some cases depending on the combination of angular velocity ratios. To solve this problem, a novel uninterrupted transmission system capable of treating various combinations of angular velocity ratios is proposed in this section.

Figure 6 shows the structure of the new uninterrupted transmission system. Although the basic structure is the same as that shown in Fig. 1, the input shaft is divided into input shafts 1 and 2, and a speed increasing/reducing device is installed between input shafts 1 and 2. In this uninterrupted transmission system, the rotation of input shaft 1 is transmitted to the output shaft through the speed increasing/reducing device and the noncircular gear when the velocity ratio is changed.

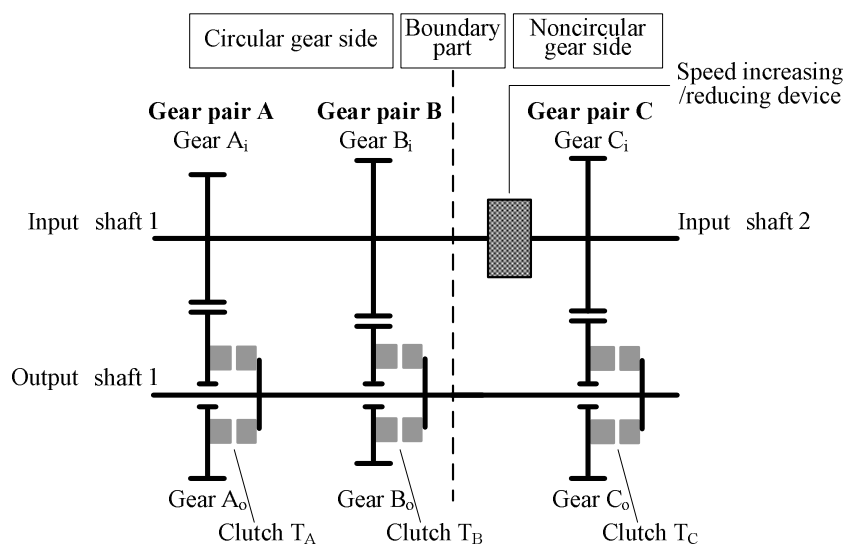

Fig. 6 Structure of uninterrupted transmission system for various combinations of angular velocity ratios

Here, the angular velocity ratio curve of this noncircular gear is shown by the dashed curve in Fig. 7. As shown in the figure, suppose that the angular velocity ratios in the constant angular velocity ratio sections of the noncircular gear are $r_{A}{ }^{\prime}$ and $r_{B}{ }^{\prime}$, and the angular velocity ratio of this speed increasing/reducing device (the value between input shaft 1 and input shaft 2 ) is $1 / K$. Figure 6 shows the boundary part between the circular gear side (left 
side) and the noncircular gear side (right side) by the dashed curve; hereafter, this part is referred to as the boundary part. The angular velocity ratio between the input and output shafts at the boundary part on the noncircular gear side is discussed here. When the noncircular gear meshes under an angular velocity ratio of $r_{A}{ }^{\prime}$, the angular velocity ratio between the input and output shafts at the boundary part is $r_{A}{ }^{\prime} / K$. On the other hand, when the noncircular gear meshes under an angular velocity ratio of $r_{B}{ }^{\prime}$, the angular velocity ratio of the input and output shafts at the boundary part is $r_{B}{ }^{\prime} / K$. These conditions are shown by the solid curve in Fig. 7.

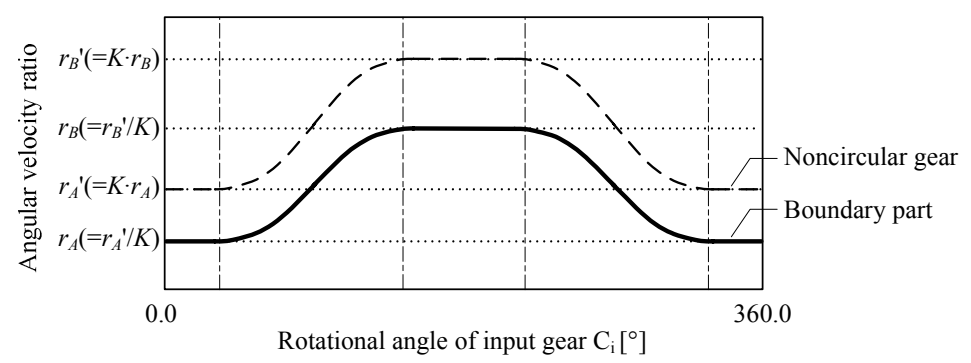

Fig. 7 Angular velocity ratio curve of noncircular gear pair $\mathrm{C}$ and boundary part

During the velocity ratio changing process of the uninterrupted transmission system, the angular velocity ratio between the input and output shafts on the circular gear side must be the same as that on the noncircular gear side at the boundary part. This condition can be expressed as follows:

$$
\begin{aligned}
& r_{A}=r_{A}{ }^{\prime} / K \\
& r_{B}=r_{B}{ }^{\prime} / K
\end{aligned}
$$

The left sides of Eqs. (1) and (2) are already-known parameters because the values of the angular velocity ratios $r_{A}$ and $r_{B}$ of the circular gear pairs are usually provided in advance. As indicated in Eqs. (1) and (2), there is a constraint between $r_{A}{ }^{\prime}$ and $r_{B}{ }^{\prime}$; the ratio between $r_{A}{ }^{\prime}$ and $r_{B}{ }^{\prime}$ must be the same as the ratio between $r_{A}$ and $r_{B}$ to satisfy Eqs. (1) and (2). On the other hand, the values of $r_{A}{ }^{\prime}$ and $r_{B}{ }^{\prime}$ can be changed by adjusting the value of $K$ while satisfying Eqs. (1) and (2). The parameters related with the design of the noncircular gear are $r_{A}{ }^{\prime}$ and $r_{B}{ }^{\prime}$. These enable flexibility in the design of the noncircular gear because the values of $r_{A}{ }^{\prime}$ and $r_{B}{ }^{\prime}$ can be changed by changing the value of $K$. The design process is as follows. First, suitable values of $r_{A}{ }^{\prime}$ and $r_{B}{ }^{\prime}$ for the noncircular gear design are selected while satisfying the constraint that the ratio between $r_{A}{ }^{\prime}$ and $r_{B}{ }^{\prime}$ is the same as the ratio between $r_{A}$ and $r_{B}$. Thereafter, the angular velocity ratio $1 / K$ of the speed increasing/reducing device is chosen based on the values of $r_{A}{ }^{\prime}$ and $r_{B}{ }^{\prime}$.

Consider here the design method for suitable values of $r_{A}{ }^{\prime}$ and $r_{B}{ }^{\prime}$ for the noncircular gear. Figure 8 shows the relationship between the angular velocity ratio curve and $K$. Take the case in which both $r_{A}{ }^{\prime}$ and $r_{B}{ }^{\prime}$ are under $1\left(r_{A}{ }^{\prime}=0.65, r_{B}{ }^{\prime}=0.85\right)$ as an example. If $K$ is $1, r_{A}$ and $r_{B}$ equal $r_{A}{ }^{\prime}$ and $r_{B}{ }^{\prime}$, respectively. In this case, the angular velocity ratio curve does not change smoothly and is not suitable for an uninterrupted transmission system. If $K$ increases to $1.25, r_{B}{ }^{\prime}$ becomes over 1 (1.0625). In this case, the angular velocity ratio curve becomes smooth, but the range of constant angular velocity ratio sections corresponding to $r_{A}{ }^{\prime}$ and $r_{B}{ }^{\prime}$ is different. If $K$ reaches 1.33, the value of 1 is near the middle position between $r_{A}{ }^{\prime}$ (0.8645) and $r_{B}{ }^{\prime}$ (1.1305). In this case, the ranges of constant angular velocity ratio sections become almost the same. Under $K=1.38, r_{A}{ }^{\prime}(0.897)$ becomes close to the value 1. In this case, the range of $r_{A}{ }^{\prime}$ 
gets long, but that of $r_{B}{ }^{\prime}$ (1.173) becomes short. In the case of $K=1.7, r_{A}{ }^{\prime}$ (1.105) becomes over 1 , and the angular velocity ratio curve does not change smoothly again. The angular velocity ratio curve changes in this way according to the value of $K$. Suitable values of $r_{A}{ }^{\prime}$ and $r_{B}{ }^{\prime}$ for a noncircular gear can be selected by understanding this change in the angular velocity ratio curve due to $K$.

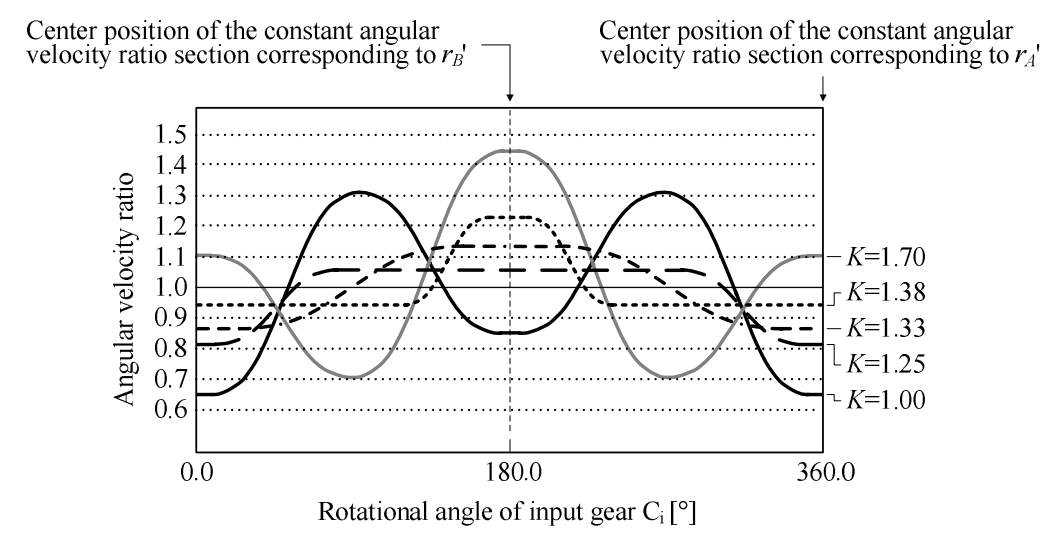

Fig. 8 Schematic model of change in angular velocity ratio curve of noncircular gear according to parameter $K$ under fixed $r_{A}$ and $r_{B}$

The selection method for suitable values of $r_{A}{ }^{\prime}$ and $r_{B}{ }^{\prime}$ for a noncircular gear design depends on the mechanical devices in which the uninterrupted transmission system is applied, and therefore, there are a variety of selection methods. An example of an ideal method is to select $r_{A}{ }^{\prime}$ and $r_{B}{ }^{\prime}$ so that the ranges of constant angular velocity ratio sections (sections a and b) become the same. In this case, $r_{A}{ }^{\prime}$ and $r_{B}{ }^{\prime}$ should be selected to satisfy the following equation:

$$
\frac{\left(r_{A}{ }^{\prime}+r_{B}{ }^{\prime}\right)}{2}=1
$$

Take the case shown in Fig. 4(a), where the uninterrupted transmission system is required to realize angular velocity ratios of 0.7 and 0.9 using this selection method. In this case, if the variable $K$ is $1.25, r_{A}{ }^{\prime}$ becomes 0.875 , and $r_{B}{ }^{\prime}$ becomes 1.125 . The noncircular gears have the angular velocity ratio curve shown by curve (a) in Fig. 9.

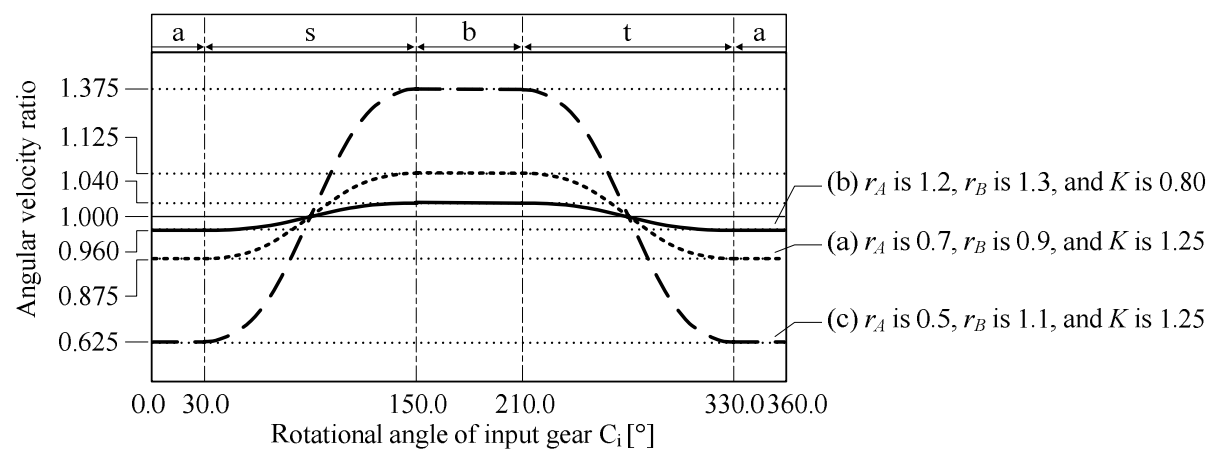

Fig. 9 Angular velocity ratio curve of noncircular gear pair C, where the proposed design method is applied

As a result, the increase and decrease of the angular velocity ratio in sections $s$ and $t$ do not occur, which is different from the state mentioned in Fig. 4(a). Curve (b) in Fig. 9 shows the angular velocity ratio curve of the noncircular gear, where the proposed design method is 
applied to the case in Fig. 4(b), in which the angular velocity $r_{A}$ is 1.2, and $r_{B}$ is 1.3. In this case, the values of the angular velocity ratios $r_{A}$ and $r_{B}$ are both larger than 1 . By selecting the value $K$ of 0.80 , it is possible to realize a suitable angular velocity ratio curve of the noncircular gear. Curve (c) in Fig. 9 shows the result for the application of the proposed design method to the case corresponding to Fig. 5, where angular velocity ratios of 0.5 and 1.1 are required. By selecting the value of $K$ to be $1.25, r_{A}{ }^{\prime}$ becomes 0.625 , and $r_{B}{ }^{\prime}$ becomes 1.375 . As a result, an ideal angular velocity ratio curve has been achieved.

In the new type of uninterrupted transmission system shown in Fig. 6, a suitable angular velocity ratio of the noncircular gear can be achieved by adjusting the parameter $K$ of the speed increasing/reducing device. In this uninterrupted transmission system, the speed increasing/reducing device can be equipped either on the input shaft side or the output shaft side, although the speed increasing/reducing device is located on the input shaft side in Fig. 6.

\section{Velocity ratio changing method to solve the constraint problem between the}

angular velocity ratio and the number of teeth on the clutches

In the uninterrupted transmission system, tooth clutches are used. As shown by the velocity ratio changing process in Fig. 3, two clutches are engaged simultaneously in sections $\mathrm{a}$ and $\mathrm{b}$. In this process, in order to engage the second clutch while the first clutch is engaged, it is necessary to match the phases of the drive side teeth to the driven side teeth on the other clutch. A theoretical analysis of the condition for clutch engagement was performed in the previous report ${ }^{(4)}$, and these conditions have been clarified. The conditions are expressed as follows:

$$
\left.\begin{array}{l}
n_{d}\left(r_{A}-r_{B}\right) \in Z \\
\frac{n_{d}\left(1-r_{A}\right)}{2} \in Z \\
\frac{n_{d}\left(1-r_{B}\right)}{2} \in Z
\end{array}\right\}
$$

where $n_{d}$ is the number of teeth on the tooth clutches, and $Z$ is a set of integers. The angular velocity ratios $r_{A}$ and $r_{B}$ of the circular gear pairs and the number of teeth $n_{d}$ on the clutch must satisfy the constraints in Eq. (4) to realize engagement of the clutches. The angular velocity ratios of the circular gear pairs determine the output characteristics of the uninterrupted transmission system. On the other hand, the number of teeth on the clutch affects the strength of the clutch. Therefore, it is desirable that they can be designed independently. However, in an uninterrupted transmission system, their design flexibility is low because the angular velocity ratio and the number of teeth on the clutch must be determined in compliance with Eq. (4).

To solve this problem, a new velocity ratio changing method for the uninterrupted transmission system is proposed in this chapter. Figure 10 shows the new type of velocity ratio changing process. The basic transition process is similar to the one shown in Fig. 3, but the timing of the clutch engagement and disengagement is different. In the previous method shown in Fig. 3, clutch $T_{A}$ is disengaged in section a after clutch $T_{C}$ is engaged. However, in the new method shown in Fig. 10, clutch $T_{C}$ is engaged after clutch $T_{A}$ is disengaged. Similarly, clutch $T_{B}$ is engaged after clutch $T_{C}$ is disengaged in section $b$ in the new method, although clutch $T_{C}$ is disengaged after clutch $T_{B}$ is engaged in the previous method. In the new method, it is possible to adjust the tooth phases of the drive side and 
driven side on the second engaged clutch since the first engaged clutch has already been disengaged.

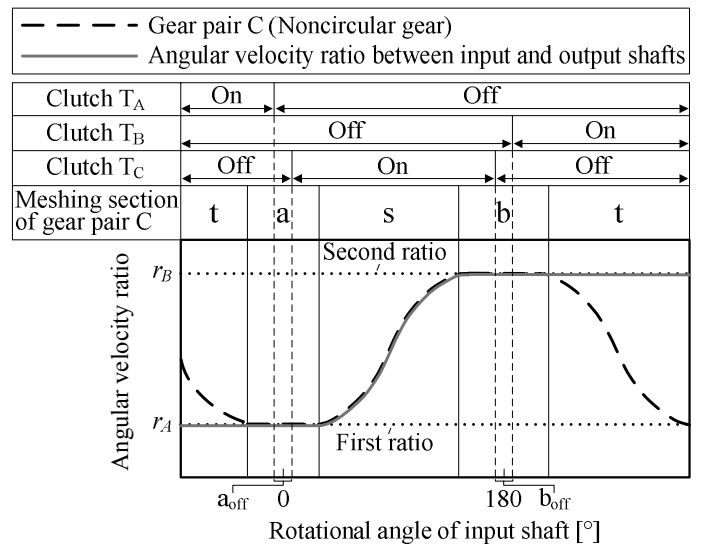

Fig. 10 Proposed transition process to change the angular velocity ratio from the first ratio to the second ratio

Figure 11(a) shows the drive and driven sides of the clutch. The clutch has triangular-shaped teeth. Suppose that the phases of the drive and driven sides of the clutch do not match before the clutch begins engagement, as shown in Fig. 11(a). In the proposed method, even if a phase difference exists, the drive side and driven side can rotate relatively. Therefore, they can eliminate the phase difference by pushing the drive side and driven side of the clutch with triangular-shaped teeth. Consequently, it is possible to engage the clutch as shown in Fig. 11(b). In this method, a phase difference in the clutch is allowed, and the constraint concerning the engagement of the tooth clutches (Eq. (4)) is not a factor. Thus, the angular velocity ratio of the circular gear pairs and the number of teeth on the clutches can be designed independently.

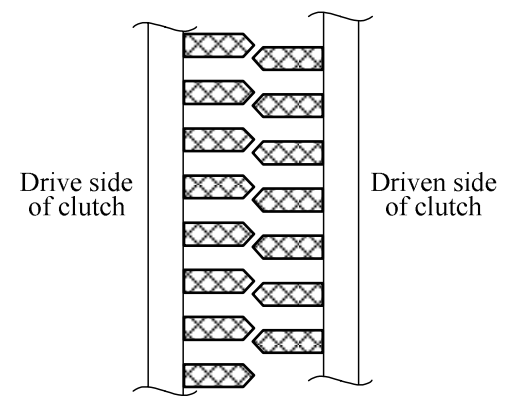

(a) Before engagement

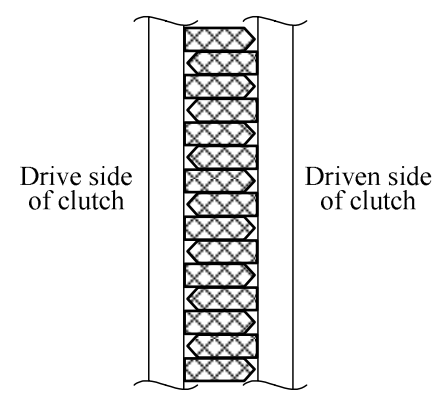

(b) After engagement

Fig. 11 Engagement of clutch under phase difference between drive side and driven side

In this method, sections $a_{\text {off }}$ and $b_{\text {off }}$ exist as shown in Fig. 10, where none of the clutches are engaged. Therefore, it is impossible to realize complete power and rotation transmission because the torque and rotational angle cannot be transmitted in these sections. However, it is possible to make the times of sections $\mathrm{a}_{\text {off }}$ and $\mathrm{b}_{\text {off }}$ short. In this uninterrupted transmission system, the circular gear pair $\mathrm{A}$ and the noncircular gear pair $\mathrm{C}$ rotate at the same rotational speed in section a; also, gear pairs $\mathrm{B}$ and $\mathrm{C}$ rotate at the same rotational speed in section $b$. Therefore, since the drive side and driven side of the clutch rotate at the same rotational speed when the clutch is engaged, the engagement can begin immediately. Thus, the durations of sections $a_{\text {off }}$ and $b_{\text {off }}$ can become short. For example, the durations of sections $a_{\text {off }}$ and $b_{\text {off }}$ are $3 \mathrm{~ms}$ in the experiment mentioned in the next chapter. If the interruption time is short, this method is considered to be useful in certain applications. 


\section{Construction of the proposed uninterrupted transmission system and} experiment

In this chapter, an experimental apparatus incorporating the proposed uninterrupted transmission system and transition method is constructed, and its effectiveness is verified by experiment.

\subsection{Experimental apparatus}

The experimental apparatus of the uninterrupted transmission system is constructed using gears from actual automotive transmissions. The tooth numbers of gear pair A (the first ratio gear) are 20 and 38, and those of gear pair B (the second ratio gear) are 33 and 34. The center distance is $99.0 \mathrm{~mm}$. The angular velocity ratios $r_{A}$ and $r_{B}$ of gear pairs A and $\mathrm{B}$ are 0.526 and 0.970 , respectively. Since they are both smaller than the natural number 1 , this condition represents the case in which it is impossible to design a suitable noncircular gear in the previous uninterrupted transmission system. This problem is solved by applying the uninterrupted transmission system proposed in $\S 3$.

The angular velocity ratio $1 / K$ of the speed increasing/reducing device is selected to be 0.748 such that the average angular velocity ratio of the noncircular gear becomes the natural number 1 . The angular velocity ratio curve of the designed noncircular gear is shown in Fig. 12. The angular velocity ratios are 0.703 and 1.296 in the constant angular velocity ratio sections (sections a and b) of the noncircular gear, and the natural number 1 is between them. Therefore, the angular velocity ratio of the noncircular gear changes smoothly in sections $s$ and $t$. This condition is different from Fig. 4(a). In section a (corresponding to the first ratio condition), the rotational angle of input gear $\mathrm{C}_{\mathrm{i}}$ is in the range from 0.0 degrees to 36.0 degrees and from 324.0 degrees to 360.0 degrees. The rotational angle in section $\mathrm{b}$ (corresponding to the second ratio condition) is in the range from 144.0 degrees to 216.0 degrees. Thus, sections a and $b$ have the same range of 72.0 degrees. The angular velocity ratio $1 / K$ of the speed increasing/reducing device is selected based on Eq. (3) such that the natural number 1 is in the middle position between the two angular velocity ratios. Because of this selection, sections $a$ and $b$ have the same range.

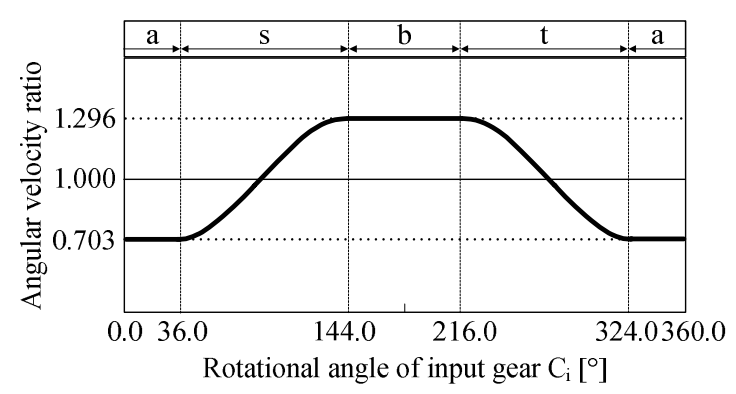

Fig. 12 Angular velocity ratio curve of noncircular gear pair $\mathrm{C}$ used in the experiment

Figure 13 shows the structure of the experimental device, and the constructed experimental apparatus is shown in Fig. 14(a). The basic structure is the same as the uninterrupted transmission system shown in Fig. 6, but the speed increasing/reducing device is equipped on the output shaft side. In Fig. 14(a), gear pairs A and B, the shafts, and clutches $\mathrm{T}_{\mathrm{A}}$ and $\mathrm{T}_{\mathrm{B}}$ on the left side of the dashed line $\mathrm{P} 1$ are actual automotive parts. The speed increasing/reducing device is made up of gear pairs D and E. The tooth numbers of gear pair D are 36 and 39, and the module is 2.75 . Those of gear pair E are 30 and 37, and the module is 3.00 . The center distance is $101.7 \mathrm{~mm}$, and the material is $\mathrm{S} 45 \mathrm{C}$.

The tooth numbers of the noncircular gear pair are 33 and 33, the module is 3.00 , the 
center distance is $99.0 \mathrm{~mm}$, and the material is S45C. Figure 14(b) shows the noncircular gear pair. The tooth numbers of clutches $T_{A}$ and $T_{B}$ are 45 , and that of clutch $T_{C}$ is 250 . These angular velocity ratios and tooth numbers of the clutch do not satisfy the constraint for tooth clutch engagement (Eq. (4)), and therefore, a problem occurs in the clutch engagement in the previous uninterrupted transmission system. This problem is solved here by applying the new transition method proposed in $\S 4$.

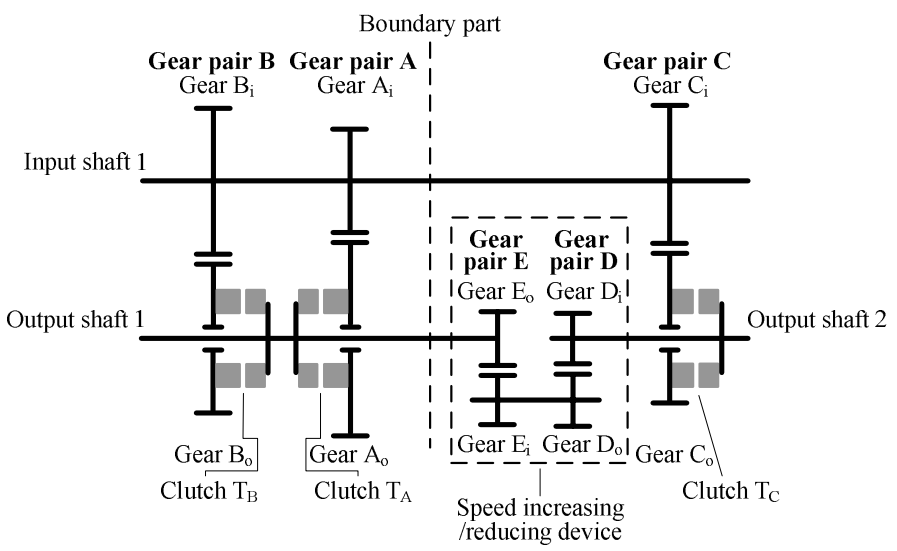

Fig. 13 Structure of experimental apparatus of uninterrupted transmission system

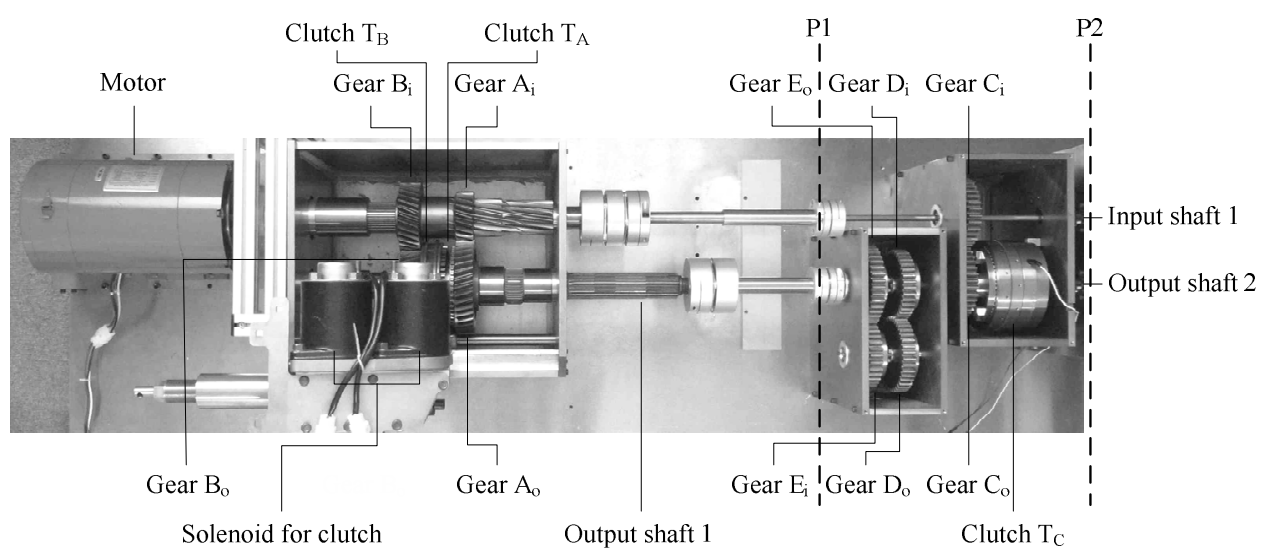

(a) Constructed uninterrupted transmission system

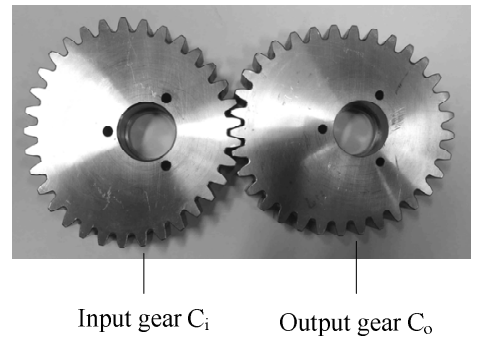

(b) Noncircular gear pair

Fig. 14 Overall view of experimental apparatus for uninterrupted transmission system and noncircular gear pair

\subsection{Measurement of the angular velocity ratio of the speed increasing/reducing device and the noncircular gear}

The combination of the speed increasing/reducing device and the noncircular gear is the main functional device in the uninterrupted transmission system proposed in $\S 3$. Its 
angular velocity ratio is measured here to verify that the angular velocity ratio changed as expected. In the experiment, the angular velocity ratio of the noncircular gear pair alone and that of the combination of the speed increasing/reducing device and the noncircular gears are both measured. In the measurement of the angular velocity ratio of the noncircular gear, rotary encoders are installed at position P2 shown in Fig. 14(a), and the measurement is performed while clutch $\mathrm{T}_{\mathrm{C}}$ is engaged. On the other hand, in the measurement of the combination of the speed increasing/reducing device and the noncircular gear, the circular gear side and the noncircular gear side are separated at position P1 in Fig. 14(a). The rotary encoders are also installed at position $\mathrm{P} 1$.

Figure 15 shows the measured angular velocity ratio. The measured angular velocity ratio curve of the noncircular gear is identical to the design curve shown in Fig. 12. The angular velocity ratios in the constant angular velocity ratio sections are around 0.70 and 1.30 , and these values are almost the same as the design values (0.703 and 1.296). On the other hand, the angular velocity ratios of the combination of the speed increasing/reducing device and the noncircular gear are about 0.52 and 0.97 in the constant angular velocity ratio sections. These values are almost the same as the angular velocity ratios of circular gear pairs A and B (0.526 and 0.970). Based on these results, it was confirmed that the angular velocity ratio can be changed as designed by using the combination of a speed increasing/reducing device and a noncircular gear.

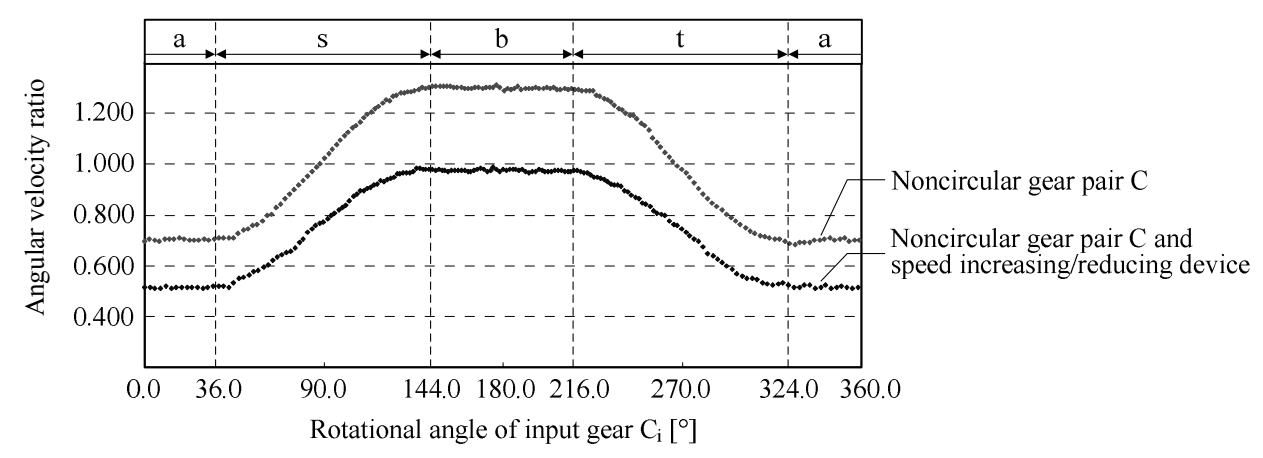

Fig. 15 Measured result of angular velocity ratio

\subsection{Experiment of velocity ratio change}

The experiment is performed using the experimental apparatus shown in Fig. 14(a) to investigate the effectiveness of the uninterrupted transmission system proposed in $\S 3$ and the transition method proposed in $\S 4$. The experiment of the velocity ratio change from the first ratio condition to the second ratio condition was performed under a constant rotational speed of $150 \mathrm{~min}^{-1}$ (input shaft speed). The experiments were conducted 10 times, and it was confirmed that the velocity ratio change was performed without any problems. Abnormal sound did not occur when clutches $\mathrm{T}_{\mathrm{A}}, \mathrm{T}_{\mathrm{B}}$, and $\mathrm{T}_{\mathrm{C}}$ engaged or disengaged.

Figure 16 shows three sets of measured angular velocity ratio data. The angular velocity ratio is calculated from the measurement data of the rotary encoders, which are installed at position P2, as shown in Fig. 14(a). The timings of the beginning and completion of operation for each clutch were set as shown in the bottom of Fig. 16(a). In the velocity ratio changing process in Fig. 10, the durations of sections $a_{\text {off }}$ and $b_{\text {off }}$, where none of the clutches are engaged, were set to $3 \mathrm{~ms}$. In clutches $\mathrm{T}_{\mathrm{A}}$ and $\mathrm{T}_{\mathrm{B}}$, it takes $60 \mathrm{~ms}$ from beginning to completion of the engagement or disengagement, and it takes $5 \mathrm{~ms}$ in clutch $\mathrm{T}_{\mathrm{C}}$.

The operation beginning point of each clutch was set based on the operation times of these clutches. In the transition from the first ratio condition to the velocity ratio changing condition, clutch $\mathrm{T}_{\mathrm{A}}$ starts the disengagement operation when the rotational angle of noncircular gear $C_{i}$ is -57.1 degrees ( 
is 7.7 degrees $\left(\right.$ step $<2>$ ). At $3 \mathrm{~ms}$ after step $<2>$, clutch $\mathrm{T}_{\mathrm{C}}$ starts the engagement at step $<3>$ (11.1 degrees), and it is completed at step $<4>$ (16.4 degrees). In the process from the velocity ratio changing condition to the second ratio condition, clutch $T_{C}$ starts the disengagement at step $<5>$ (152.3 degrees), and it is completed at step $<6>$ (155.9 degrees). Clutch $\mathrm{T}_{\mathrm{B}}$ starts the engagement at step $<7>$ (158.3 degrees) $3 \mathrm{~ms}$ later, and it is completed at step $<8>$ (202.5 degrees). In all of the results in (a), (b), and (c) in Fig. 16, the measured angular velocity ratio changed smoothly from 0.703 (the angular velocity ratio of the first ratio) to 1.296 (that of the second ratio) for the designed angular velocity ratio of the noncircular velocity ratio shown in Fig. 12. No significant decrease in the angular velocity ratio appeared during the engagement operation of the clutches. Thus, it is confirmed that the clutch engagement was achieved without any problems.

(a)

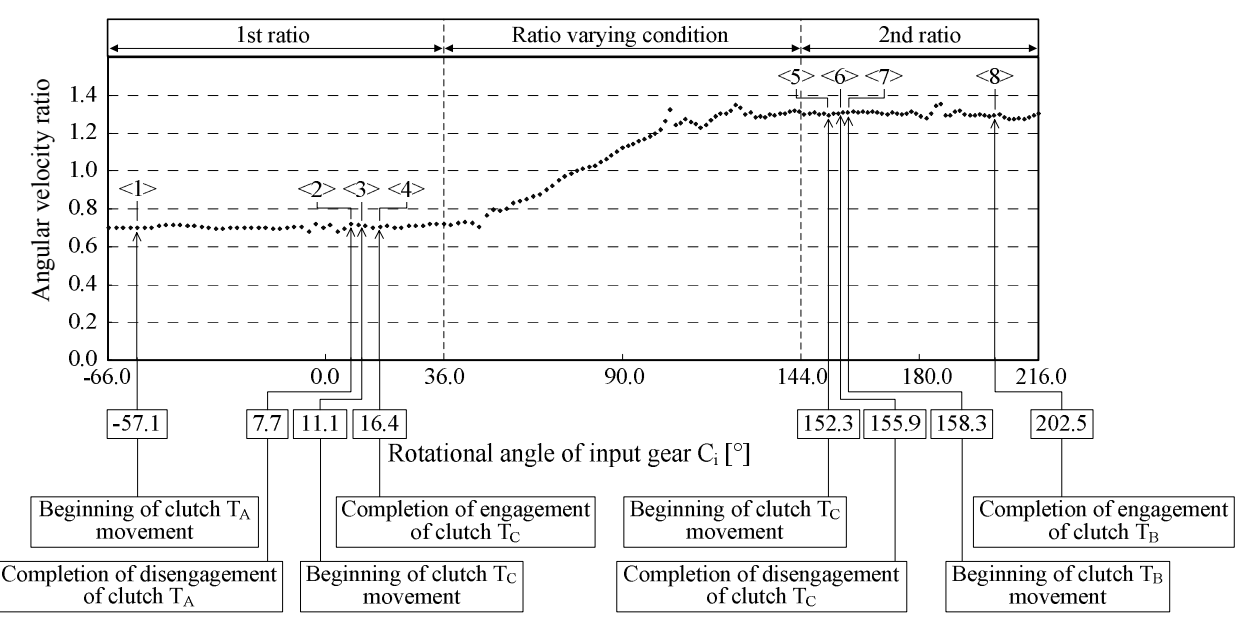

(b)

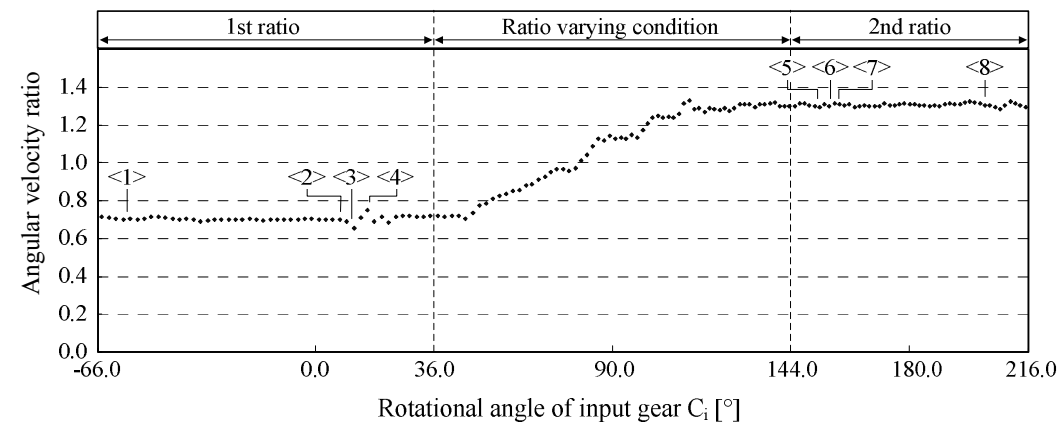

(c)

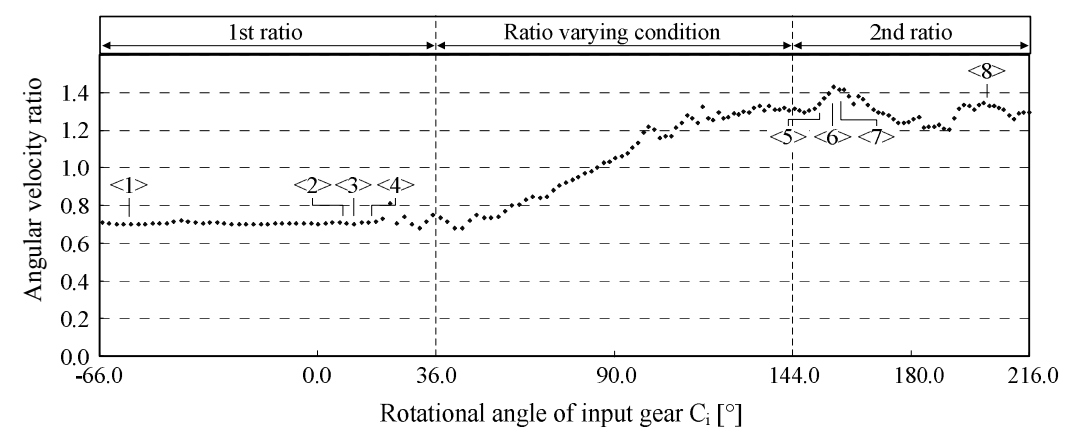

Fig. 16 Experimental results when the angular velocity ratio is changed from the first ratio to the second ratio

Next, observe the sections from $<2>$ to $<3>$ and from $<6>$ to $<7>$, where no clutch is engaged. In the section from $<2>$ to $<3>$, the change in the angular velocity ratio is little in all of the measured results. The angular velocity ratio does not change much either in the section from $<6>$ to $<7>$. In the sections from $<2>$ to $<3>$ and from $<6>$ to $<7>$, there was a 
possibility that the decrease in the angular velocity ratio appears since the torque cannot be transmitted because none of the clutches are engaged. However, the interruption time is short (3 ms), and therefore, it is considered that the angular velocity ratio did not change.

Now observe the section from $<3>$ to $<4>$, where clutch $\mathrm{T}_{\mathrm{C}}$ is engaged. No significant change in the angular velocity ratio is observed in (a) and (c), but the angular velocity ratio increases in (b). When clutch $\mathrm{T}_{\mathrm{C}}$ starts to engage, the phases of the drive side and driven side of clutch $T_{C}$ does not match in some cases. The engagement operation starts from this state, the triangular-shaped teeth of clutch $T_{C}$ eliminate the phase difference between the drive side and driven side of the clutch, and then the clutch is engaged. Thus, it is considered that the angular velocity ratio between the input and output shafts has changed slightly in (b).

Next, observe the section from $<7>$ to $<8>$, where clutch $T_{B}$ is engaged. No significant change is observed in (a) or (b). However, the angular velocity ratio decreases in (c). As mentioned above, this change in the angular velocity ratio is caused by the elimination of the phase difference between the drive side and driven side of clutch $\mathrm{T}_{\mathrm{B}}$. On the other hand, there are some cases in which a change in the angular velocity ratio was not observed during the velocity ratio changing process. In the section from $<3>$ to $<4>$ in Fig. 16(a) and (c), where clutch $T_{C}$ is engaged, and the section from $<7>$ to $<8>$ in Fig. 16(a) and (b), where clutch $T_{B}$ is engaged, the angular velocity ratio did not change. The states of the phase difference between the drive side and driven side of the clutch vary when the clutch starts the engagement operation. It is considered that the phase difference between the drive side and driven side of the clutch did not exist or was slight in cases where the angular velocity ratio did not change.

Based on these results, it is confirmed that the clutch can be engaged using the proposed transition method (even if there is a phase difference in the clutch) and that there is a possibility of temporary changes in the angular velocity ratio at clutch engagement. Consequently, the experimental results verified that the uninterrupted transmission system proposed in $\S 3$ and the transition method proposed in $\S 4$ are effective.

\section{Conclusion}

The uninterrupted transmission system using the noncircular gear proposed in the previous reports can transmit torque and precise rotational angles during the velocity ratio changing process. However, it is difficult to design a suitable noncircular gear under some angular velocity ratio conditions. In addition, it is impossible to design the gears and clutches independently since there is a constraint to be satisfied between the angular velocity ratio and the number of teeth on the clutch.

In this research, a new type of uninterrupted transmission system was proposed that can accommodate various angular velocity ratios. A new transition method was also proposed that solves the constraint problem between the angular velocity ratio and the number of teeth on the clutch and increases the design flexibility. The following results were obtained:

(1) If a certain natural number does not fall between the angular velocity ratios required for the transmission, it is difficult to design the noncircular gear. To solve this problem, an uninterrupted transmission system using the combination of a speed increasing/reducing device and noncircular gears was proposed. It was possible to realize the angular velocity ratios required for the transmission system while achieving angular velocity ratios suitable for the noncircular gear design by adjusting the angular velocity ratio of the speed increasing/reducing device. Moreover, this method makes it possible to equalize the range corresponding to the constant angular velocity ratio sections of the noncircular gear. 
(2) A new transition method was proposed where two clutches are simultaneously not engaged (two clutches are temporarily disengaged at the same time) in the constant angular velocity ratio sections of the noncircular gear. By using this method and the clutch with triangular-shaped teeth, engagement operations can be realized even if a phase difference exists between the drive side and driven side of the clutch. Therefore, it is possible to avoid the constraint between the angular velocity ratio and the number of teeth on the clutch. This leads to high flexibility in the design.

(3) An experimental apparatus was constructed based on the proposed uninterrupted transmission system and the transition method using gears and clutches from actual automobiles. The design condition evaluated in this experiment caused problems concerning the design of the noncircular gear and the clutches in the previous uninterrupted transmission system. The experimental results showed that a suitable noncircular gear can be achieved and smooth changes in the angular velocity ratio can be realized using the new uninterrupted transmission system. Furthermore, it confirmed that the proposed transition method makes it possible to realize clutch engagement without any problems even if a phase difference exists in the clutch. Consequently, the experimental results verified that the new uninterrupted transmission system is capable of accommodating various combinations of angular velocity ratios and clutch tooth numbers.

\section{Acknowledgement}

This study was supported by Industrial Technology Research Grant Program in 2009 from New Energy and Industrial Technology Development Organization (NEDO) of Japan.

\section{References}

(1) Nozaki, Y., Tanaka, Y., Tomomatsu, H., Tsukamoto, H., Hanji, F., “Toyota's New Six-Speed Automatic Transmission A761E for RWD Vehicles", SAE Technical Paper, 2004-01-0650(2004).

(2) Gama Frorecio, D., Resis Assis, E., Ferreira Amendola, C., "The Manual Transmission Automated - Gearshift Quality Comparison to a Similar Manual System", SAE Technical Paper, 2004-01-3363(2004).

(3) Tseng, C., Hsieh, M., "Analysis and Optimization of Clutch Actuator on Automated Manual Transmission System”, SAE Technical Paper, 2005-01-1782(2005).

(4) Komori, M., Kang, J., Kimura, Y., "Study on the Ratio Variable system to Transmit Power and Motion Continuously", Journal of Japan society for design engineering, Vol.45, No.10(2010), pp.512-519, (in Japanese).

(5) Komori, M., Kang, J., Takeoka, F., Kimura, Y., Onodera, Y., "Geometrical Analysis of Noncircular Gear for Ratio Variation System and Modifying Method of Ratio Variation", Journal of Japan society for design engineering, Vol.46, No.7(2011), pp.402-409, (in Japanese).

(6) Kang, J., Komori, M., Takeoka, F., Onodera, Y., "Multi-Speed Ratio Variable System to Transmit Power and Motion Continuously", Transactions of the Japan Society of Mechanical Engineers, Series C, Vol.77, No.782(2011), pp.329-338, (in Japanese).

(7) Kang, J., Komori, M., Zhang, S., Sugiyama, K., "Control Method for Output Speed during Velocity Ratio Change under High Rotational Speed Using an Uninterrupted Transmission System", Journal of Advanced Mechanical Design, Systems, and Manufacturing, Vol.6, No.7(2012), pp.1281-1297. 